\title{
RESEARCH
}

Open Access

\section{Insights into globalization: comparison of patient characteristics and disease progression among geographic regions in a multinational Alzheimer's disease clinical program}

Jeffrey L. Cummings ${ }^{1 *}$, Alireza Atri ${ }^{2,3}$, Clive Ballard ${ }^{4}$, Neli Boneva ${ }^{5}$, Lutz Frölich ${ }^{6}$, José Luis Molinuevo ${ }^{7,8}$, Lars Lau Raket ${ }^{5}$ and Pierre N. Tariot ${ }^{9}$

\begin{abstract}
Background: Globalization of clinical trials has important consequences for trial planning and interpretation. This study investigated heterogeneity in patient characteristics and outcomes among world regions in the global idalopirdine Phase 3 clinical program.

Methods: Data were pooled from three 24-week randomized controlled trials in patients aged $\geq 50$ years with mild-to-moderate Alzheimer's disease (AD) $(n=2506)$. Patients received idalopirdine $(10,30$, or $60 \mathrm{mg} /$ day) or placebo, added to cholinesterase inhibitor treatment. Patients were categorized into the following regions: Eastern Europe/Turkey $(n=759)$, Western Europe/Israel $(n=709)$, USA/Canada $(n=444)$, South America/Mexico $(n=361)$, Asia $(n=134)$, and Australia/South Africa $(n=99)$. For each region, operational characteristics, baseline demographic and clinical characteristics, adverse events, and mean change from baseline to week 24 in clinical rating scale scores (placebo group only) were summarized using descriptive statistics.

Results: Completion rates were $0.86-0.90$ in all regions. Heterogeneity among global regions was evident. Protocol deviations were twice as common in South America/Mexico as in USA/Canada (2.64 vs 1.35 per patient screened). Educational level ranged from 9.2 years in South America/Mexico to 13.4 years in USA/Canada. APOE $\varepsilon 4$ carriage was $80.6 \%$ in Australia/South Africa, $63.1 \%$ in Western Europe/Israel, and $<60 \%$ in other regions. Screening Mini-Mental State Examination scores were higher in Eastern Europe/Turkey (18.0) and USA/Canada (17.5) than in other regions (16.9-17.1). Baseline AD Assessment Scale—Cognitive subscale (ADAS-Cog) scores ranged from 24.3 in USA/Canada to 27.2 in South America/Mexico. Baseline AD Cooperative Study_Activities of Daily Living, 23-item version (ADCS-ADL 23 ) scores ranged from 58.5 in USA/Canada to 53.5 in Eastern Europe/Turkey. In the placebo group, adverse events were 1.6-1.7 times more common in Western Europe/Israel, USA/Canada, and Australia/South Africa than in Eastern Europe/ Turkey. On the ADAS-Cog, Australia/South Africa and Western Europe/lsrael showed the most worsening among patients receiving placebo (1.56 and 1.40 points, respectively), whereas South America/Mexico showed an improvement (-0.71 points). All regions worsened on the $A D C S-A_{23}$, from -3.21 points in Western Europe/Israel to -0.59 points in Eastern Europe/Turkey.

(Continued on next page)
\end{abstract}

\footnotetext{
* Correspondence: cumminj@ccf.org

${ }^{1}$ Cleveland Clinic Lou Ruvo Center for Brain Health, 888 W Bonneville

Avenue, Las Vegas, NV 89106, USA

Full list of author information is available at the end of the article
}

(c) The Author(s). 2018 Open Access This article is distributed under the terms of the Creative Commons Attribution 4.0 International License (http://creativecommons.org/licenses/by/4.0/), which permits unrestricted use, distribution, and reproduction in any medium, provided you give appropriate credit to the original author(s) and the source, provide a link to the Creative Commons license, and indicate if changes were made. The Creative Commons Public Domain Dedication waiver (http://creativecommons.org/publicdomain/zero/1.0/) applies to the data made available in this article, unless otherwise stated. 
(Continued from previous page)

Conclusions: Regional heterogeneity — in terms of study conduct, patient characteristics, and outcomes-exists, and should be accounted for, when planning and conducting multinational AD clinical trials.

Trial registration: ClinicalTrials.gov, NCT01955161. Registered on 27 September 2013. ClinicalTrials.gov, NCT02006641. Registered on 5 December 2013. ClinicalTrials.gov, NCT02006654. Registered on 5 December 2013.

Keywords: Alzheimer's disease, Humans, Patient selection, Globalization, Clinical trial, Dementia, Disease progression, Cognitive dysfunction, Cognitive decline, Activities of daily living

\section{Background}

Alzheimer's disease (AD) is a highly prevalent, age-related, degenerative brain disease, associated with considerable burden for patients, their family members, caregivers, and society [1]. As the global elderly population increases, the costs associated with $\mathrm{AD}$ are also expected to rise [1]. Currently, only two classes of drug are approved for the treatment of AD: cholinesterase inhibitors (ChEIs) and an $N$-methyl-D-aspartate (NMDA) receptor antagonist, memantine $[2,3]$. While these agents, and their combination, can provide patients with a symptomatic benefit [2, 4], there remains an urgent need for additional treatment options that can prevent $\mathrm{AD}$, delay its onset, slow its progression, or improve its symptoms [5].

Increasingly, clinical trials of potential new drugs in $\mathrm{AD}$ are conducted on an international basis, which allows for faster recruitment of patients, has lower costs, and provides the opportunity to pursue registration and sales in different regions [6, 7]. However, in a global trial, heterogeneity may emerge among geographic regions in terms of clinical characteristics, demographics, comorbidities, clinical measures, and side-effect profiles $[6,8]$. This heterogeneity may arise due to differences among regions in pharmacokinetics and pharmacogenetics, the culture of diagnosis and care, the translation of study materials, the experience of study sites, and the attitudes of patients and investigators toward clinical studies $[6,8]$.

The extent to which these regional factors can impact clinical study outcomes, and to which they should be considered in the design of multinational studies, is unknown. Using data from the multinational Phase 3 clinical program of idalopirdine, the aim of this analysis was to understand the effect of globalization on AD clinical trial design and outcomes by comparing study conduct, patient completion rates, patient characteristics, adverse events, and disease progression across regions.

\section{Methods}

\section{Study design and patients}

Data were pooled from three 24-week, randomized, double-blind, placebo-controlled studies in mild-tomoderate AD: STARSHINE (ClinicalTrials.gov NCT01 955161), STARBEAM (ClinicalTrials.gov NCT020066
41), and STARBRIGHT (ClinicalTrials.gov NCT020 06654). In these studies, treatment with idalopirdinea selective serotonin $5-\mathrm{HT}_{6}$ receptor antagonist-did not reduce cognitive loss compared with placebo over 24 weeks [9]. For a full description of the designs and outcomes of these studies, see Atri et al. [9].

Each of the three studies had a similar design, comprising: a 2-week screening period during which patient eligibility was monitored by qualified medical staff employed by the sponsor; a 24-week, randomized, double-blind treatment period with regular safety and efficacy assessments; and a 4-week safety follow-up period (or enrollment into an open-label extension study). Patients were enrolled from 34 countries. The studies included male and female outpatients aged $\geq 50$ years with a National Institute of Neurological and Communicative Disorders and StrokeAlzheimer's Disease and Related Disorders Association (NINCDS-ADRDA) criteria diagnosis of probable AD [10], with a Mini-Mental State Examination (MMSE) score of 12-22 at screening [11], and who had received a therapeutic and stable dose of ChEI for at least 4 months prior to screening (donepezil in STARSHINE and STARBEAM, any ChEI in STARBRIGHT). Patients were excluded if they were taking memantine, had an alternative cause of dementia, had serious non-AD central nervous system or somatic disorders, had clinically significant abnormalities as determined by laboratory testing, or were taking concomitant medications that would interfere with the safety and efficacy assessments. Eligible patients were randomized to double-blind treatment with idalopirdine (fixed doses of 10, 30 , or $60 \mathrm{mg} /$ day, depending on the study) or placebo, taken in addition to their standard ChEI treatment.

\section{Outcome measures}

The primary outcome measure of each study was the AD Assessment Scale-Cognitive subscale (ADAS-Cog), an 11-item, objective measure of cognitive impairment scored from 0 to 70 , with a higher score indicating more impairment [12]. Key secondary outcome measures in each study were the AD Cooperative Study-Activities of Daily Living, 23-item version (ADCS-ADL $\mathrm{AL}_{23}$ ) and the AD Cooperative Study-Clinical Global Impression of Change (ADCS-CGIC). The ADCS- $\mathrm{ADL}_{23}$ is an informant- 
rated measure of functional impairment scored from 0 to 78 , where a higher score indicates less impairment $[13,14]$. The ADCS-CGIC is a clinician-rated measure of: global severity at baseline scored from 1 (normal, not at all ill) to 7 (among the most extremely ill patients); and global change at follow-up scored from 1 (marked improvement) to 7 (marked worsening), where 4 indicates no change $[15,16]$. Other secondary outcome measures included the Neuropsychiatric Inventory (NPI) and the MMSE. The NPI is an informant-rated measure of behavioral disturbance (administered by the clinician) scored from 0 to 144, where a higher score indicates more disturbance [17]. The MMSE is an objective measure of cognitive impairment scored from 0 to 30 , where a higher score indicates less impairment [11]. External quality oversight methods, including central review of scale administration, were used to achieve consistent and accurate ratings throughout the studies for the ADAS-Cog, ADCS$\mathrm{ADL}_{23}, \mathrm{ADCS}-\mathrm{CGIC}$, and MMSE.

Safety was assessed via the reporting of adverse events, classified according to the Medical Dictionary for Regulatory Activities (MedDRA) version 19.0.

\section{Data analysis}

For the present post-hoc analysis, patients were categorized into geographic regions according to shared culture, history, geography, and linguistic features, based on the work of Glickman et al. [7] and previous regional studies of AD $[18,19]$, adjusted for the regions included in the idalopirdine global development program. The resulting six geographic regions were: Eastern Europe/Turkey (comprising Bulgaria, Croatia, Czech Republic, Estonia, Hungary, Lithuania, Poland, Romania, Serbia, Slovakia, Turkey, and Ukraine); Western Europe/Israel (comprising Belgium, Denmark, Finland, France, Germany, Israel, Italy, Portugal, Spain, Switzerland, and United Kingdom); USA/Canada (comprising Canada and USA); South America/Mexico (comprising Argentina, Brazil, Chile, and Mexico); Asia (comprising South Korea, Singapore, and Taiwan); and Australia/South Africa (comprising Australia and South Africa).

For each region, operational characteristics of the clinical program were summarized using descriptive statistics, including the number of sites that screened at least one patient and that randomized at least one patient, the number of patients screened and number of patients randomized per month, and the randomization, completion, and protocol deviation rates.

Baseline demographic and clinical characteristics were summarized descriptively by region for the all-patients-treated set (APTS), defined as all randomized patients who took at least one dose of double-blind medication. Statistical comparisons of baseline variables across all regions were calculated using Kruskal-Wallis tests for the continuous variables, and chi-squared tests for the categorical variables.

The incidences of adverse events, serious adverse events, and deaths were summarized descriptively, per patient in the APTS, by treatment group and by region.

Mean change from baseline to week 24 in the clinical rating scale scores was summarized descriptively using observed cases, by region, in the subpopulation of patients who were randomized to placebo and completed their respective study. For the ADCS-CGIC, which is itself a measure of change from baseline, the mean absolute value at week 24 was summarized using observed cases. Clinical outcomes were investigated in the placebo group rather than the total population to make the results more generalizable, and to prevent the bias that may occur due to a drug's specific mode of action in relation to the genetic and environmental differences in patients across regions. Statistical comparisons of equality of variances were calculated using Levene tests, and pairwise comparisons between specific regions were calculated using Tukey tests. In case the assumption of equal variances was violated, sensitivity analyses based on Games-Howell post-hoc tests were performed. To evaluate whether regional differences in observed change from baseline to week 24 in the clinical rating scale scores were driven by regional differences in educational level, a linear regression analysis that adjusted for both region and years of education was used as a sensitivity analysis.

Testing was done using a 0.05 significance level (two-sided) with no overall correction for multiple comparisons (regions were corrected for in the Tukey and Games-Howell tests). The statistical analyses were performed using SAS version 9.4 (SAS Institute Inc.).

\section{Results \\ Study conduct}

Operational characteristics of the clinical program across regions are presented in Table 1. Western Europe/Israel and USA/Canada had the greatest number of sites (135 and 132, respectively), whereas Asia and Australia/South Africa had the fewest sites (27 and 16, respectively). In Asia and USA/Canada, approximately a third of sites were 'minimal recruiters' (randomizing 0-1 patients), whereas in Eastern Europe/Turkey only $3.6 \%$ of sites were minimal recruiters. Overall, randomization rates were highest in Eastern Europe/Turkey (0.69 patients randomized per patient screened) and lowest in Asia and USA/Canada (0.51 and 0.48 patients randomized per patient screened, respectively). Completion rates were high in all regions, in the range of $0.86-0.90$ completers per patient randomized. Protocol deviation rates were highest in South America/Mexico (2.64 protocol deviations per patient screened) and lowest in USA/ Canada (1.35 protocol deviations per patient screened). 
Table 1 Operational characteristics of the clinical program by region

\begin{tabular}{|c|c|c|c|c|c|c|}
\hline & $\begin{array}{l}\text { Eastern } \\
\text { Europe } \\
\text { /Turkey }\end{array}$ & $\begin{array}{l}\text { Western } \\
\text { Europe } \\
\text { /Israel }\end{array}$ & $\begin{array}{l}\text { USA } \\
\text { /Canada }\end{array}$ & $\begin{array}{l}\text { South America } \\
\text { /Mexico }\end{array}$ & Asia & $\begin{array}{l}\text { Australia } \\
\text { /South Africa }\end{array}$ \\
\hline Sites that screened at least 1 patient & 83 & 135 & 132 & 45 & 27 & 16 \\
\hline Randomized 0 patients & 1 & 6 & 19 & 1 & 5 & 2 \\
\hline Randomized 1 patient only & 2 & 14 & 23 & 2 & 4 & 2 \\
\hline Randomized 0-1 patients (\%) & $3(3.6)$ & $20(14.8)$ & $42(31.8)$ & $3(6.7)$ & $9(33.3)$ & $4(25.0)$ \\
\hline Patients screened per month ${ }^{a}$ & 1.23 & 1.78 & 1.41 & 2.03 & 2.07 & 1.98 \\
\hline Patients randomized per month ${ }^{\mathrm{b}}$ & 0.84 & 1.22 & 0.58 & 1.30 & 0.92 & 0.98 \\
\hline Randomization rate $^{c}$ & 0.69 & 0.64 & 0.48 & 0.66 & 0.51 & 0.54 \\
\hline Completion rate $^{d}$ & 0.89 & 0.90 & 0.89 & 0.88 & 0.90 & 0.86 \\
\hline Protocol deviation rate ${ }^{\mathrm{e}}$ & 1.79 & 2.33 & 1.35 & 2.64 & 1.47 & 1.44 \\
\hline
\end{tabular}

${ }^{\mathrm{a}}$ Mean of values for each site (number of patients screened/duration of screening period in months). Screening period was defined as the time from first patient screened to last patient screened at the site; sites that screened patients for a period of $<7$ days were assigned a screening period of 7 days for this calculation ${ }^{\mathrm{b}}$ Mean of values for each site (number of patients randomized/duration of screening period in months). Screening period was defined as the time from first patient screened to last patient screened at the site; sites that screened patients for a period of $<7$ days were assigned a screening period of 7 days for this calculation

'Mean of values for each site (number of patients randomized/number of patients screened)

${ }^{\mathrm{d}}$ Total number of patients completed across all sites/total number of patients randomized across all sites

${ }^{\mathrm{e}}$ Mean of values for each site (number of protocol deviations throughout the study/number of patients screened)

\section{Demographics}

A total of 2506 patients received at least one dose of randomized treatment, split across the regions as follows: Eastern Europe/Turkey, 759 (30.3\% of the total); Western Europe/Israel, 709 (28.3\%); USA/Canada, 444 (17.7\%); South America/Mexico, 361 (14.4\%); Asia, 134 (5.3\%); and Australia/South Africa, 99 (4.0\%).

Baseline demographics by region are presented in Table 2. In summary, regional differences were observed for height, weight, body mass index (BMI), educational level, marital status, relationship of caregiver, apolipoprotein $\mathrm{E}(A P O E) \varepsilon 4$ carriage, and MMSE score (all $p<$ 0.001 ), but not for age or sex. Specifically, height was lowest in Asia $(1.57 \mathrm{~m})$ and South America/Mexico $(1.59 \mathrm{~m})$, and comparable in the other regions (1.63$1.64 \mathrm{~m})$. Weight and BMI were lowest in Asia $(57.6 \mathrm{~kg}$, $\left.23.4 \mathrm{~kg} / \mathrm{m}^{2}\right)$, and highest in Australia/South Africa $\left(71.9 \mathrm{~kg}, 26.8 \mathrm{~kg} / \mathrm{m}^{2}\right)$ and USA/Canada $(71.6 \mathrm{~kg}, 26.6 \mathrm{~kg} /$ $\left.\mathrm{m}^{2}\right)$. Educational level was lowest in South America/ Mexico (9.2 years) and Asia (9.6 years), and highest in USA/Canada (13.4 years). The proportion of married patients was lowest in Eastern Europe/Turkey (58.2\%) and highest in Asia (77.6\%). Most commonly, caregivers were a spouse or partner in Western Europe/Israel, USA/ Canada, Asia, and Australia/South Africa, and a child in Eastern Europe/Turkey and South America/Mexico. $A P O E$ \&4 carriage was most common in Australia/South Africa (80.6\%), followed by Western Europe/Israel $(63.1 \%)$, and was $<60 \%$ in the other regions, being lowest in Asia (51.5\%).

At screening, the MMSE score was higher (indicating less cognitive impairment) in Eastern Europe/Turkey (18.0) and USA/Canada (17.5) than in the other regions (range 16.9-17.1). A similar pattern was seen at baseline. All regions showed a mean improvement in the MMSE score from screening to baseline. The degree of improvement ranged from 0.5 points in Eastern Europe/ Turkey to 0.9 points in USA/Canada, South America/ Mexico, and Australia/South Africa. For complete comparisons, see Table 2 .

\section{Clinical characteristics}

Clinical characteristics by region are presented in Table 2 . Overall, regional differences were observed for time since AD diagnosis, prestudy treatment duration, previous treatment with a ChEI, and previous treatment with memantine (all $p<0.0001$ ). Median time since AD diagnosis varied from 1.3 years in Eastern Europe/Turkey to 2.2 years in Australia/South Africa. Median prestudy treatment duration varied from 1.0 year in Eastern Europe/Turkey to 1.3 years in Australia/South Africa and USA/Canada. Previous treatment with a ChEI other than donepezil (among patients currently treated with donepezil as required by protocol) ranged from $2.4 \%$ in Eastern Europe/Turkey to $7.8 \%$ in Western Europe/Israel. Previous treatment with memantine ranged from $1.0 \%$ in Australia/South Africa to $20.0 \%$ in USA/Canada.

\section{Baseline clinical rating scale scores}

Regional differences at baseline, also presented in Table 2, were observed for the ADAS-Cog, ADCSCGIC, and ADCS- $\mathrm{ADL}_{23}$ (all $p<0.0001$ ), but not for the NPI. The baseline ADAS-Cog score was lowest (less cognitive impairment) in USA/Canada (24.3) and highest (more cognitive impairment) in South America/Mexico (27.2). The baseline ADCS-CGIC (severity) scores were 


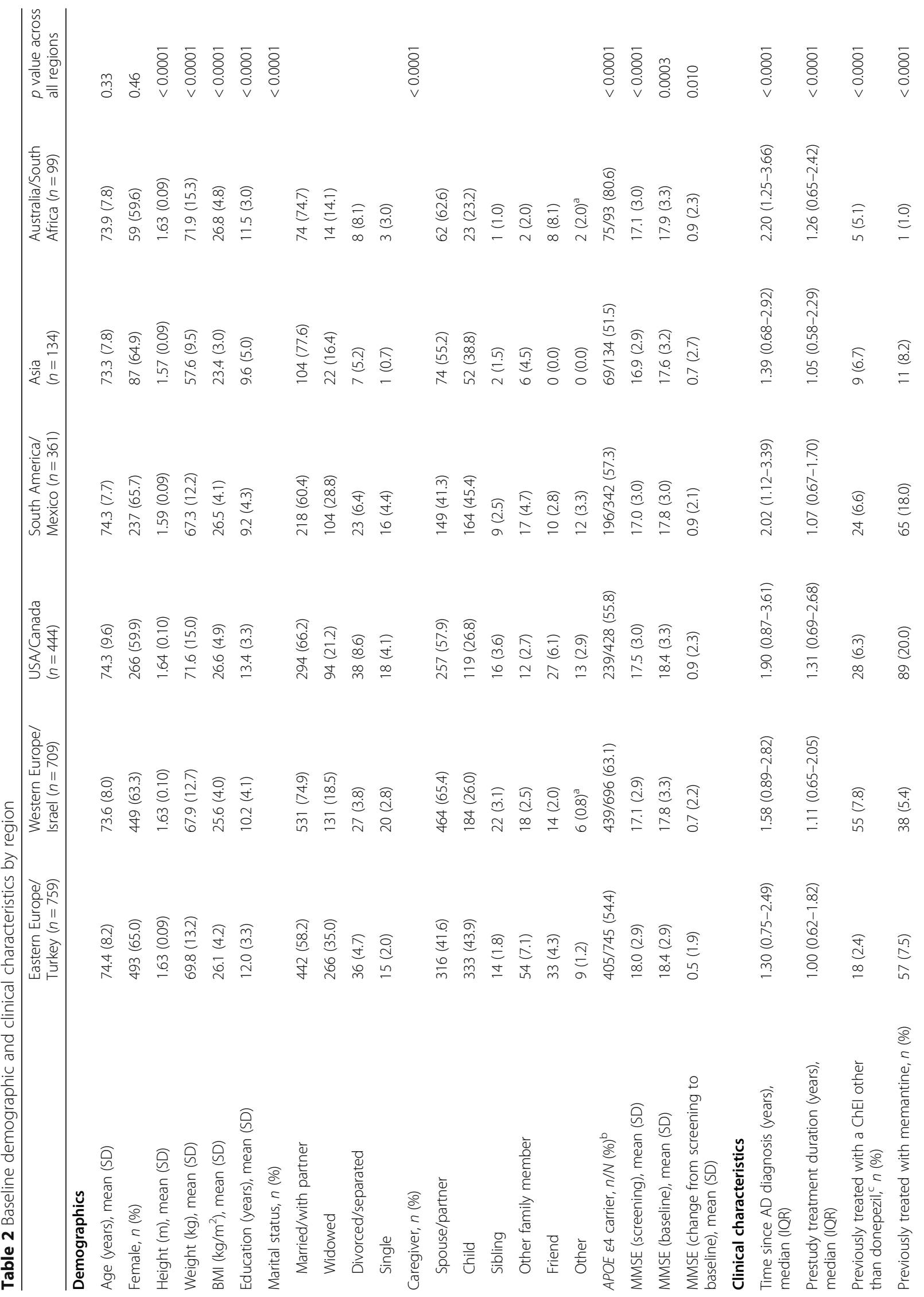




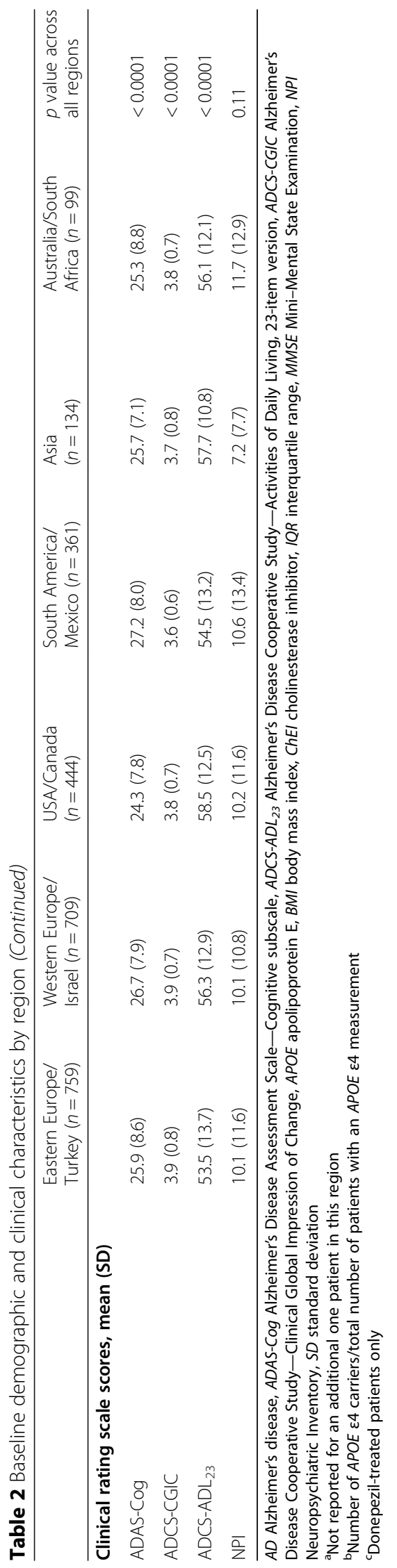


lowest (less severely ill) in South America/Mexico (3.6) and Asia (3.7), and highest (more severely ill) in Eastern Europe/Turkey and Western Europe/Israel (both 3.9). The baseline ADCS-ADL ${ }_{23}$ score was highest (less functional impairment) in USA/Canada (58.5), and lowest (more functional impairment) in Eastern Europe/Turkey (53.5). Although not statistically different across all groups, the baseline NPI score was lower (less behavioral disturbance) in Asia (7.2) than in the other regions (10.1-11.7).

\section{Adverse events}

The incidence of adverse events by region is presented in Table 3. The lowest incidence of adverse events was in Eastern Europe/Turkey, and the highest incidences were generally in Western Europe/Israel, USA/Canada, and Australia/South Africa. In the placebo group, for example, adverse events were 1.6-1.7 times more common in Western Europe/Israel, USA/ Canada, and Australia/South Africa than in Eastern Europe/Turkey. The incidence of serious adverse events, and of deaths, was low and comparable in all regions.

\section{Observed change from baseline to week 24 in the placebo group}

Mean changes in the clinical rating scale scores from baseline to week 24 in the placebo group are presented in Table 4 and Fig. 1. On the ADAS-Cog, all regions showed worsening except for South America/Mexico, which showed an improvement $(-0.71)$. The largest changes from baseline were seen in Australia/South Africa (1.56) and Western Europe/Israel (1.40). Regional differences were also observed on the global outcome (ADCS-CGIC) at week 24: Western Europe/Israel (mean score 4.51), Australia/South Africa (4.41), and USA/ Canada (4.39) showed the most worsening, whereas South America/Mexico (3.98) and Eastern Europe/ Turkey (4.18) showed no change or the least worsening. Comparison of variance across regions revealed a difference for ADCS-CGIC $(p<0.0001)$, thus violating the assumptions of the Tukey test. A sensitivity analysis assuming unequal variances across regions (GamesHowell post-hoc test) confirmed the significant pairwise differences, except for USA/Canada and South America/ Mexico (adjusted $p=0.075$ ).

All regions showed worsening on the functioning outcome (ADCS-ADL 23 ); the most worsening was seen in Western Europe/Israel (-3.21), and the least in Eastern Europe/Turkey (-0.59). The behavioral outcome (NPI) showed improvement in all regions (range -0.13 to -1.73 ) except for Western Europe/Israel (0.45). The MMSE score worsened in all regions (range -0.34 to -1.22 ) except for Eastern Europe/Turkey (0.10).
When adjustments were made for education as well as region, years of education predicted a faster rate of decline on the ADAS-Cog (0.16 points per added year of education, $p=0.0024)$, and the adjusted difference between Western Europe/Israel and South America/ Mexico remained significant. Years of education was not a significant predictor for rate of decline for the other endpoints (all $p>0.2$ ).

\section{Discussion}

This analysis demonstrated considerable heterogeneity among geographic regions in the multinational idalopirdine Phase 3 clinical program in AD. Despite the uneven distribution of patients across regions, with small proportions of the total population in Asia and Australia/ South Africa, heterogeneity was evident with regard to study conduct, baseline demographic and clinical characteristics, the incidence of adverse events, and the progression of disease in the placebo group. Differences were evident despite all global sites using identical study protocols.

Considering baseline demographic and clinical characteristics, there was a 14-kg difference between the regions with the greatest mean weight (USA/Canada and Australia/South Africa) and the region with the lowest mean weight (Asia). BMI followed a similar regional pattern. Weight and BMI are important considerations in clinical trial design, since they can affect drug distribution and clearance [20] and may affect brain exposure of the administered agent. Patients in USA/Canada had, on average, around 4 years' more education than patients in South America/Mexico and Asia. A greater level of education has been linked to faster cognitive decline in $\mathrm{AD}$ $[21,22]$, which was observed in this analysis on the ADAS-Cog. The increased rate of decline is thought to arise as a consequence of 'cognitive reserve', referring to the ability of some people to tolerate greater neuropathology without developing clinical symptoms [23]. Cognitive reserve is enhanced by education and, therefore, patients with a higher level of education have a greater degree of pathology before clinical symptoms of the disease become manifest [23]. Since the speed of disease progression increases with disease severity, these same patients may deteriorate more rapidly upon diagnosis [21, 22].

Other than Australia/South Africa (80.6\%), all regions had a lower proportion of $A P O E \& 4$ carriers than has been observed in populations of biologically proven $\mathrm{AD}$ patients (around 70\% in the EXPEDITION3 trial) [24]. This could imply that non-AD patients were recruited into the program, particularly at sites outside Australia and South Africa. Alternatively, these differences could reflect racial differences in the frequency of $A P O E \varepsilon 4$, which is higher in black populations than white populations $[25,26]$. The relationship 


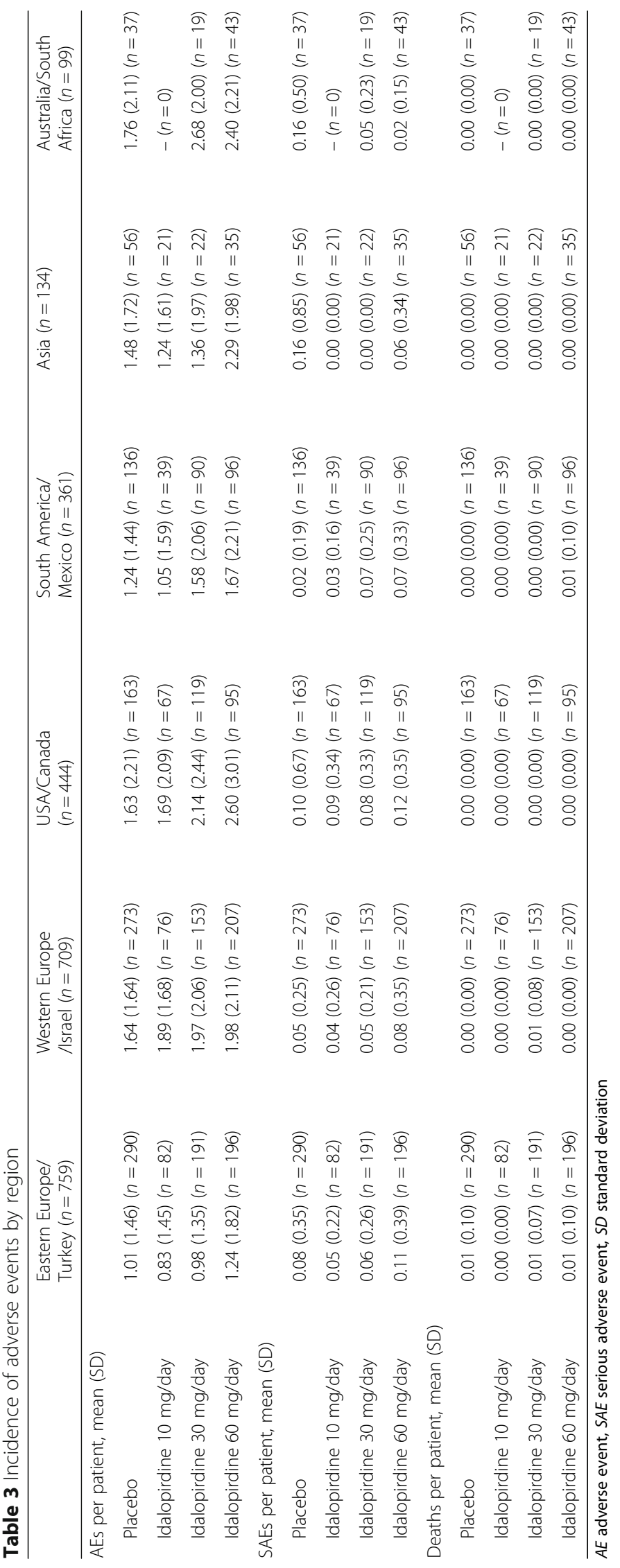




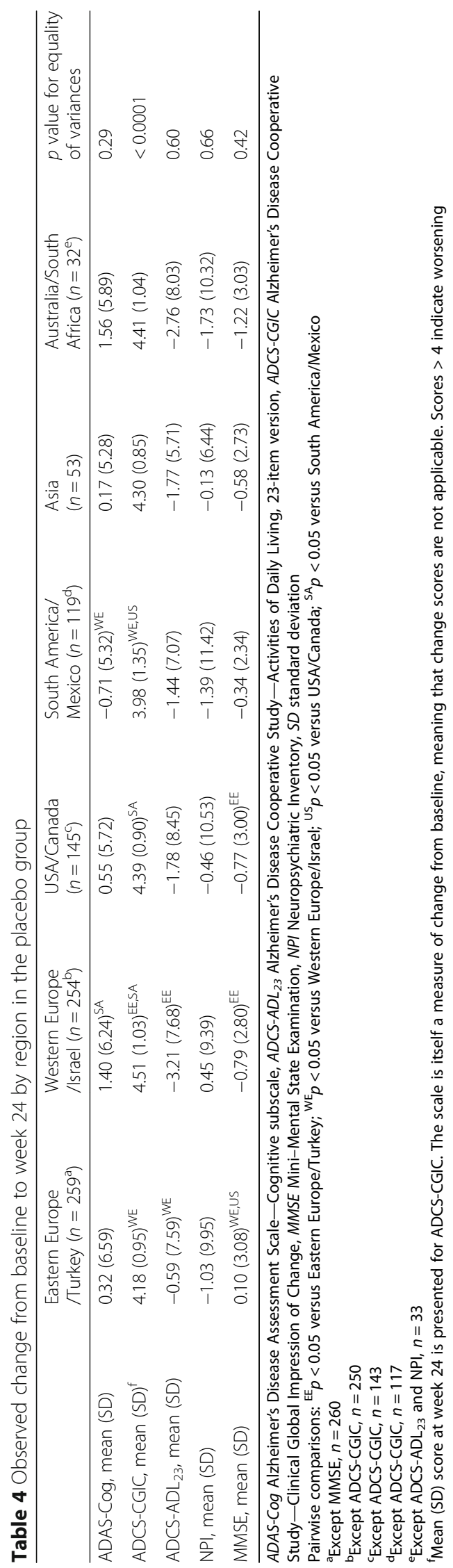


ADAS-Cog

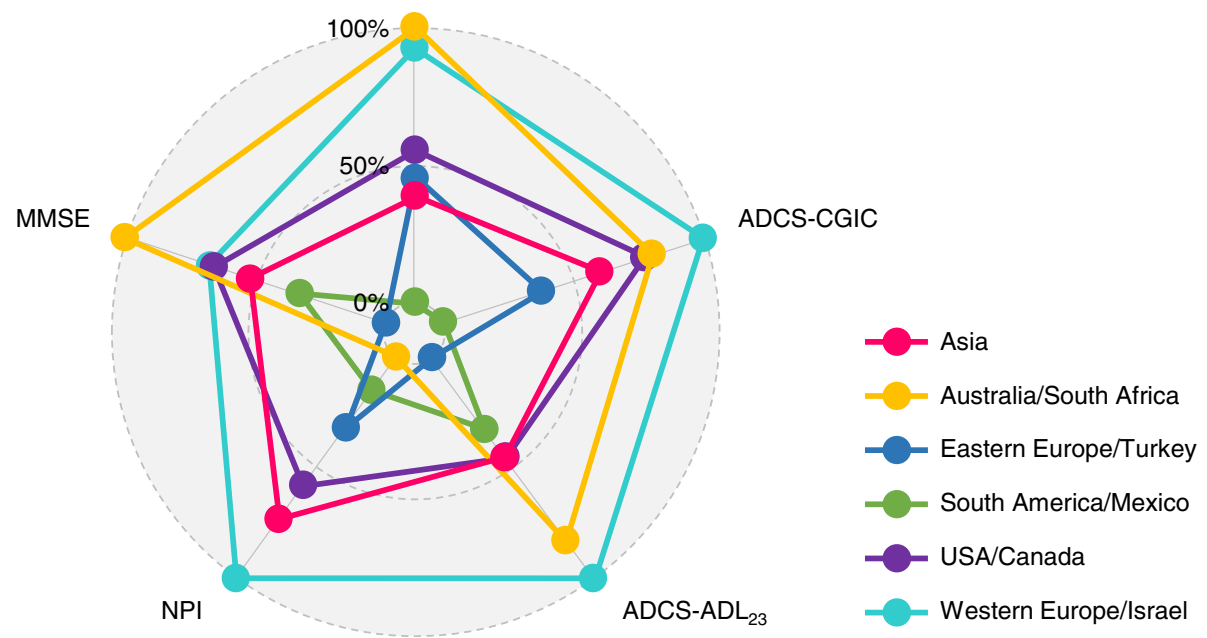

Fig. 1 Normalized observed decline from baseline to week 24 across regions in placebo group. 100\%, maximal decline on the scale observed across regions; 0\%, minimum decline/maximum improvement observed on the scale across regions. ADAS-Cog Alzheimer's Disease Assessment Scale-Cognitive subscale, $A D C S-A D L_{23}$ Alzheimer's Disease Cooperative Study-Activities of Daily Living, 23-item version, ADCS-CG/C Alzheimer's Disease Cooperative Study - Clinical Global Impression of Change, MMSE Mini-Mental State Examination, NPI Neuropsychiatric Inventory

between $A P O E \& 4$ status and risk of late-onset $\mathrm{AD}$ also varies among races, being strongest and most well established in European and Asian populations [27, 28].

Several differences in diagnosis and treatment history were observed across regions, which may reflect differences in culture, societal awareness of $\mathrm{AD}$, and standard of care. The time since AD diagnosis was almost a year longer in Australia/South Africa than in Eastern Europe/ Turkey; this could suggest that patients in Eastern Europe/Turkey present to the clinic later in the disease (although MMSE scores at screening were not lower in Eastern Europe/Turkey, indicating a discrepancy between the two approaches to severity), or that study inclusion criteria (such as the requirement for stable donepezil/ChEI treatment) selected for patients with a greater disease duration in some regions.

Considering patient treatment history prior to enrollment, use of a ChEI other than donepezil (among those currently treated with donepezil) was low in all regions $(<10 \%)$, whereas prior memantine treatment varied from around 20\% in USA/Canada and South America/Mexico to $<10 \%$ in all other regions. In the USA, memantine is often initiated as early as ChEIs, despite memantine not being approved for use in mild disease [29]. In the South America/Mexico group, memantine use was mainly driven by patients from Argentina, where reimbursement policy has historically favored memantine such that it has become the most prescribed anti-dementia drug [30]. Of note, the idalopirdine study entry criteria excluded patients currently receiving memantine, which may have contributed to inclusion of more atypical patients in regions where memantine use is common. The reasons why patients stopped memantine treatment prior to the start of the study were not collected.

Baseline clinical rating scale scores were heterogeneous among regions despite the fact that patients were monitored for eligibility during the 2-week screening period, and despite standardization of clinical rating scale usage. There were also differences in patient stability during the screening period, as shown by variation in the change in MMSE score from the screening visit to the baseline visit. Overall, at baseline, patients were least cognitively impaired in USA/Canada, and most impaired in South America/Mexico. Patients in USA/Canada also had the least impairment of functioning at baseline. The greatest impairment in functioning was observed in Eastern Europe/Turkey, which could reflect reporting behaviors of the different informants in this region, with a low proportion of married patients and with more patients being cared for by a child rather than by a spouse or partner. The suitability of activity of daily living (ADL) items to elderly patients differs across regions and can potentially affect functional metrics [31, 32]. Finally, patients showed numerically less behavioral disturbance in Asia than in other regions. Neuropsychiatric symptoms are known to vary among cultures, being influenced by lack of recognition, misinterpretation of certain behaviors, and social stigma [33-36].

Based on analysis of the change in clinical rating scale scores over the first 6 months in the placebo group, the greatest decline (on ADAS-Cog, ADCS-CGIC, and $\mathrm{ADCS}-\mathrm{ADL}_{23}$ ) was observed in Western Europe/Israel 
and Australia/South Africa, and the least decline was observed in Eastern Europe/Turkey and South America/ Mexico. The variations may reflect differences in standard of care or accuracy of diagnosis among regions, as well as the proportion of $A P O E \& 4$ carriers. Such variations in placebo decline will influence the outcome of clinical trials, since low rates of decline among patients receiving placebo will make it more difficult to distinguish a treatment effect.

Overall, two regions stood out in their patterns of characteristics at screening/baseline and their subsequent outcomes/trajectories: Eastern Europe/Turkey and South America/Mexico. Eastern Europe/Turkey had the most impaired functioning $\left(\mathrm{ADCS}-\mathrm{ADL}_{23}\right.$ ) at baseline as well as the least worsening in functioning, which could reflect: the low proportion who were married and differences between informants, as already discussed; cultural differences in the appreciation of the importance of different ADLs; and the positive effect on quality of life and standard of care that can be achieved from enrollment in a clinical trial. This latter point may also be reflected in the observation that Eastern Europe/Turkey was the only region to show improvement on the MMSE over 24 weeks in the placebo group. With regard to South America/Mexico, a similar pattern to that of Eastern Europe/Turkey was observed in terms of baseline $\mathrm{ADCS}-\mathrm{ADL}_{23}$, change in $\mathrm{ADCS}-\mathrm{ADL}_{23}$, and proportion of married patients. In addition, South America/Mexico was the only region to show improvement on the ADAS-Cog over 24 weeks (placebo group), despite having the most cognitive impairment at baseline; this may be linked to the lower level of education in this region and differing approaches to diagnosis.

The studies involved in this analysis used a centralized review of scale administration, which reduces administration errors and deviations. Studies not using centralized rating may exhibit more variability.

The incidence of adverse events across treatment arms tended to be higher in Western Europe/Israel, USA/ Canada, and Australia/South Africa than in other regions. In the idalopirdine $60 \mathrm{mg} /$ day group, for example, patients in USA/Canada experienced on average one more adverse event than patients in Eastern Europe/ Turkey and South America/Mexico. This may have been influenced by differences in baseline medical status (i.e., comorbidities) across regions. Awareness of such variations in adverse event reporting is important for industry sponsors of drug development programs, since reports from Eastern Europe and South America may not reflect the side-effect profile seen in other global regions.

Other large multinational $\mathrm{AD}$ clinical programs (e.g., semagacestat and solanezumab) have also shown heterogeneity among geographic regions $[18,19]$. Compared with the idalopirdine clinical program, the patterns of heterogeneity were generally similar with regard to baseline demographic and clinical characteristics, with, for example, weight being highest in USA/Canada and Australia/South Africa, and lowest in Asia (and Japan, a separate region in [18]); patient functioning being worst in Eastern Europe and best in USA/ Canada; patients showing less behavioral disturbance in Asia (and Japan in [18]) than in other regions; and the incidence of adverse events across treatment arms tending to be higher in USA/Canada and Australia/South Africa than in other regions. Completion rates were high and similar in all regions in the idalopirdine clinical program, whereas significant differences were found among regions in the semagacestat and solanezumab programs. The idalopirdine studies were considerably shorter ( 24 weeks) than the semagacestat/solanezumab studies (76-80 weeks), so it is possible that differences in completion rates would have emerged given a longer study duration. Solanezumab requires monthly infusions, which may also have influenced participant retention. Furthermore, there may be differences between trials of a symptomatic agent versus a potentially disease-modifying agent.

Heterogeneity may arise for many reasons, including differences in culture and standard of care for patients with AD. A heterogeneous patient population can be advantageous in a clinical program in order to show how an investigative agent affects a real-world $A D$ population. However, it is possible that a heterogeneous patient population may indicate that patients without $\mathrm{AD}$ or with atypical $\mathrm{AD}$ are being included, making it more difficult to observe a treatment effect, and highlighting the importance in future $\mathrm{AD}$ dementia trials of recruiting patients with biomarker evidence of amyloid pathology and neurodegeneration, if proposed research diagnostic criteria are validated.

This analysis of a large clinical trial program is limited by its post-hoc nature, since these studies were not designed to assess regional differences. In addition, homogeneity was not tested within the defined geographic regions, the population sizes were small in the Asia and Australia/South Africa groups, and the analysis did not control for multiple comparisons. These analyses are intended to provide observations of potential importance to those planning, conducting, and interpreting global clinical trials.

\section{Conclusions}

These analyses-conducted on a large, global, clinical trial program-demonstrated that regional heterogeneity exists in multinational $\mathrm{AD}$ clinical trials, and that it should be accounted for, whether by limiting or excluding certain countries, increasing sample size to account for the variance, or finding additional means of increasing homogeneity (e.g., biomarkers). Sponsors must be familiar with regional differences in order to appropriately plan and power studies of global programs. Differences in training and vigilance may also be required among regions. 


\section{Abbreviations}

AD: Alzheimer's disease; ADAS-Cog: Alzheimer's Disease Assessment Scale-Cognitive subscale; ADCS-ADL 23 : Alzheimer's Disease Cooperative Study_Activities of Daily Living, 23-item version; ADCS-CGIC: Alzheimer's Disease Cooperative Study_Clinical Global Impression of Change; ADL: Activity of daily living; AE: Adverse event; APOE: Apolipoprotein E; APTS: All-patients-treated set; BMI: Body mass index; ChEl: Cholinesterase inhibitor; IQR: Interquartile range; MedDRA: Medical Dictionary for Regulatory Activities; MMSE: Mini-Mental State Examination; NINCDS-ADRDA: National Institute of Neurological and Communicative Disorders and StrokeAlzheimer's Disease and Related Disorders Association; NMDA: N-methyl-Daspartate; NPI: Neuropsychiatric Inventory; SAE: Serious adverse event; SD: Standard deviation

\section{Acknowledgements}

Writing support was provided by Chris Watling, PhD, assisted by his colleagues at Cambridge Medical Communication Ltd (Cambridge, UK), and funded by H. Lundbeck A/S.

The authors acknowledge the contributions of the principal investigators in the original studies; the Idalopirdine Development Teams; the STAR Program Teams, operational partners, and site staff and investigators; and those individuals serving on the data and safety monitoring board from the respective trials. In addition, they express gratitude for the commitment of the study participants and their caregivers, without whose generous contributions and dedication this research would not be possible.

\section{Funding}

This research was supported by funding from $\mathrm{H}$. Lundbeck A/S. H. Lundbeck A/S was responsible for the design and conduct of the study; the collection, management, and analysis of data; and aided in the interpretation of data and the writing of the manuscript.

\section{Availability of data and materials}

$\mathrm{H}$. Lundbeck A/S provides secure access to anonymized patient-level data to qualified researchers in response to scientifically valid research proposals. Further details can be found online (http://www.lundbeck.com/trials).

\section{Authors' contributions}

$J L C, A A, C B, L F, J L M$, and PNT served as global, European, or US coordinating principal investigators for the idalopirdine Phase 3 STAR clinical trial program. All authors contributed to the concept and design of the post-hoc analysis. LLR analyzed the data. All authors were involved in interpreting the data, drafting the manuscript, and providing critical revision of the manuscript for intellectual content. All authors read and approved the final manuscript.

\section{Ethics approval and consent to participate}

All studies were conducted in accordance with the International Conference on Harmonisation Good Clinical Practice Guideline and the Declaration of Helsinki. Local ethics committees approved all aspects of study design. Eligible patients or their legal representatives provided written informed consent prior to the start of the studies.

\section{Consent for publication}

Not applicable.

\section{Competing interests}

JLC reported serving as a consultant to AbbVie, Acadia, Actinogen, Adamas, Alzheon, Anavex, Astellas, Avanir, Avid, Axovant, Boehringer Ingelheim, Bracket, Eisai, GE Healthcare, Genentech, Intra-Cellular Therapies, Lilly, H. Lundbeck A/S, MedAvante, Merck, Neurocog, Novartis, Orion, Otsuka, Pfizer, Piramal, QR Pharma, reMYND, Resverlogix, Roche, Suven, Takeda, Toyama, and Transition; receiving research support from Avid and Teva; owning stock options in Prana, Neurokos, Adamas, MedAvante, and QR Pharma; and owning the copyright of the Neuropsychiatric Inventory and all its derivatives. JLC is supported by Keep Memory Alive and a COBRE grant from the NIGMS (P20GM109025).

AA reported receiving honoraria for consulting, participating in independent data safety monitoring boards, providing educational lectures, programs, and materials, or serving on advisory boards for Allergan, the Alzheimer's Association, Axovant, Biogen, Grifols, Harvard Medical School Graduate Continuing Education, Lundbeck, Merck, Roche/Genentech, Sunovion, and
Suven; receiving book royalties from Oxford University Press; and having institutional contracts or receiving investigational clinical trial-related funding from the American College of Radiology, AbbVie, Avid, Biogen, Lilly, Lundbeck, Merck, and vTV.

CB reported receiving research grants from Acadia and H. Lundbeck A/S; and receiving honoraria from Acadia, Bristol-Myers Squibb, Heptares, Lilly, H. Lundbeck A/S, Novartis, Orion, Otsuka, and Roche.

NB and LLR are full-time employees of H. Lundbeck A/S.

LF reported receiving honoraria for consulting, providing educational lectures, materials, and programs, or serving on advisory boards for Avid-Eli Lilly \& Co, Avraham Pharmaceuticals, Axon Neuroscience, Boehringer Ingelheim, GE Healthcare, H. Lundbeck A/S, Merck Sharpe \& Dohme, Novartis, Nutricia, Pfizer, Piramal Imaging, Schwabe Pharma, TAD Pharma, and Takeda.

JLM reported receiving honoraria for consulting, providing educational lectures, or serving on advisory boards for $A B L$, Axovant, Boehringer Ingelheim, Eli Lilly \& Co, Fujirebio, GE Healthcare, H. Lundbeck A/S, Merck Sharpe \& Dohme, Novartis, Pfizer, Piramal Imaging, Roche, and Roche Diagnostics.

PNT reported receiving consulting fees from Abbott Laboratories, AbbVie, AC Immune, Auspex, Boehringer Ingelheim, Clintara, CME Inc., Corium, GliaCure, INSYS, and T3D; receiving consulting fees and research support from AstraZeneca, Avanir, Cognoptix, Lilly, H. Lundbeck A/S, Merck and Company, and Takeda; receiving research support from Elan, Functional Neuromodulation, Genentech, Novartis, Roche, Targacept, the National Institute on Aging, and the Arizona Department of Health Services; owning stock options in Adamas; and being listed as a contributor to a patent owned by the University of Rochester.

No other disclosures were reported.

\section{Publisher's Note}

Springer Nature remains neutral with regard to jurisdictional claims in published maps and institutional affiliations.

\section{Author details}

${ }^{1}$ Cleveland Clinic Lou Ruvo Center for Brain Health, 888 W Bonneville Avenue, Las Vegas, NV 89106, USA. ²Banner Sun Health Research Institute, Sun City, AZ, USA. 'Brigham and Women's Hospital and Harvard Medical School, Boston, MA, USA. ${ }^{4}$ University of Exeter Medical School, Exeter, UK. ${ }^{5} \mathrm{H}$. Lundbeck A/S, Valby, Denmark. ${ }^{6}$ Central Institute of Mental Health, Medical Faculty Mannheim, University of Heidelberg, Heidelberg, Germany. ${ }^{7}$ Alzheimer's Disease and Other Cognitive Disorders Unit, IDIBAPS, Hospital Clinic i Universitari, Barcelona, Spain. ${ }^{8}$ Barcelonaßeta Brain Research Center, Pasqual Maragall Foundation, Barcelona, Spain. ${ }^{9}$ Banner Alzheimer's Institute, Phoenix, AZ, USA.

Received: 19 July 2018 Accepted: 24 October 2018

Published online: 24 November 2018

References

1. Alzheimer's Association. 2016 Alzheimer's disease facts and figures. Alzheimers Dement. 2016;12:459-509.

2. Atri A. Effective pharmacological management of Alzheimer's disease. Am J Manag Care. 2011;17(Suppl 13):S346-55

3. Parsons CG, Danysz W, Dekundy A, Pulte I. Memantine and cholinesterase inhibitors: complementary mechanisms in the treatment of Alzheimer's disease. Neurotox Res. 2013;24:358-69.

4. Atri A, Rountree SD, Lopez OL, Doody RS. Validity, significance, strengths, limitations, and evidentiary value of real-world clinical data for combination therapy in Alzheimer's disease: comparison of efficacy and effectiveness studies. Neurodegener Dis. 2012;10:170-4.

5. Cummings JL, Morstorf T, Zhong K. Alzheimer's disease drug-development pipeline: few candidates, frequent failures. Alzheimers Res Ther. 2014;6:37.

6. Cummings J, Reynders R, Zhong K. Globalization of Alzheimer's disease clinical trials. Alzheimers Res Ther. 2011;3:24.

7. Glickman SW, McHutchison JG, Peterson ED, Cairns CB, Harrington RA, Califf RM, Schulman KA. Ethical and scientific implications of the globalization of clinical research. N Engl J Med. 2009;360:816-23.

8. International Conference on Harmonisation $(\mathrm{ICH})$. Ethnic factors in the acceptability of foreign clinical data. London: Guideline E5(R1); 1998. 
9. Atri A, Frölich L, Ballard C, Tariot PN, Molinuevo JL, Boneva N, et al. Effect of idalopirdine as adjunct to cholinesterase inhibitors on change in cognition in patients with Alzheimer disease: three randomized clinical trials. JAMA. 2018;319:130-42.

10. McKhann G, Drachman D, Folstein M, Katzman R, Price D, Stadlan EM. Clinical diagnosis of Alzheimer's disease: report of the NINCDS-ADRDA Work Group under the auspices of Department of Health and Human Services Task Force on Alzheimer's Disease. Neurology. 1984;34:939-44.

11. Folstein MF, Folstein SE, McHugh PR. "Mini-mental state". A practical method for grading the cognitive state of patients for the clinician. J Psychiat Res. 1975;12:189-98.

12. Rosen WG, Mohs RC, Davis KL. A new rating scale for Alzheimer's disease. Am J Psychiatry. 1984;141:1356-64.

13. Galasko D, Bennett D, Sano M, Ernesto C, Thomas R, Grundman M, Ferris S. The Alzheimer's Disease Cooperative Study. An inventory to assess activities of daily living for clinical trials in Alzheimer's disease. Alzheimer Dis Assoc Disord. 1997;11(Suppl 2):S33-9.

14. Robert P, Ferris S, Gauthier S, Ihl R, Winblad B, Tennigkeit F. Review of Alzheimer's disease scales: is there a need for a new multi-domain scale for therapy evaluation in medical practice? Alzheimers Res Ther. 2010;2:24.

15. Schneider LS, Olin JT, Doody RS, Clark CM, Morris JC, Reisberg B, et al. The Alzheimer's Disease Cooperative Study. Validity and reliability of the Alzheimer's Disease Cooperative Study_Clinical Global Impression of Change. Alzheimer Dis Assoc Disord. 1997;11(Suppl 2):S22-32.

16. Guy W. ECDEU assessment manual for psychopharmacology, revised. Rockville: National Institute of Mental Health; 1976.

17. Cummings JL. The Neuropsychiatric Inventory: assessing psychopathology in dementia patients. Neurology. 1997;48(Suppl 6):S10-6.

18. Grill JD, Raman R, Ernstrom K, Aisen P, Dowsett SA, Chen YF, et al. Comparing recruitment, retention, and safety reporting among geographic regions in multinational Alzheimer's disease clinical trials. Alzheimers Res Ther. 2015;7:39.

19. Henley DB, Dowsett SA, Chen YF, Liu-Seifert H, Grill JD, Doody RS, et al. Alzheimer's disease progression by geographical region in a clinical trial setting. Alzheimers Res Ther. 2015;7:43.

20. Hanley MJ, Abernethy DR, Greenblatt DJ. Effect of obesity on the pharmacokinetics of drugs in humans. Clin Pharmacokinet. 2010;49:71-87.

21. Meng X, D'Arcy C. Education and dementia in the context of the cognitive reserve hypothesis: a systematic review with meta-analyses and qualitative analyses. PLoS One. 2012;7:e38268.

22. Thomas RG, Albert M, Petersen RC, Aisen PS. Longitudinal decline in mildto-moderate Alzheimer's disease: analyses of placebo data from clinical trials. Alzheimers Dement. 2016:12:598-603.

23. Stern Y. Cognitive reserve in ageing and Alzheimer's disease. Lancet Neurol. 2012;11:1006-12.

24. Honig LS, Vellas B, Woodward M, Boada M, Bullock R, Borrie M, et al. Trial of solanezumab for mild dementia due to Alzheimer's disease. N Engl J Med. 2018;378:321-30.

25. Kuller LH, Shemanski L, Manolio T, Haan M, Fried L, Bryan N, et al. Relationship between ApoE, MRI findings, and cognitive function in the Cardiovascular Health Study. Stroke. 1998;29:388-98.

26. Teruel BM, Rodríguez JJ, McKeigue P, Mesa TC, Fuentes E, Cepero AV, et al. Interactions between genetic admixture, ethnic identity, APOE genotype and dementia prevalence in an admixed Cuban sample; a cross-sectional population survey and nested case-control study. BMC Med Genet. 2011:12:43.

27. Farrer LA, Cupples LA, Haines JL, Hyman B, Kukull WA, Mayeux R, et al. for the APOE and Alzheimer Disease Meta-Analysis Consortium. Effects of age sex, and ethnicity on the association between apolipoprotein E genotype and Alzheimer disease. A meta-analysis. JAMA. 1997;278:1349-56.

28. Sadigh-Eteghad S, Talebi M, Farhoudi M. Association of apolipoprotein E epsilon 4 allele with sporadic late onset Alzheimer's disease. A metaanalysis. Neurosciences (Riyadh). 2012;17:321-6.

29. Koller D, Hua T, Bynum JPW. Treatment patterns with anti-dementia drugs in the United States: Medicare cohort study. J Am Geriatr Soc. 2016;64:1540-8.

30. Rojas G, Demey I, Arizaga RL. Medicamentos utilizados para trastornos cognitivos. Análisis de un millón y medio de prescripciones en la Argentina. Medicina (B Aires). 2013;73:213-23.

31. Senanarong $V$, Harnphadungkit $K$, Prayoonwiwat N, Poungvarin N, Sivasariyanonds $\mathrm{N}$, Printarakul $\mathrm{T}$, et al. A new measurement of activities of daily living for Thai elderly with dementia. Int Psychogeriatr. 2003;15:135-48.
32. Chiu HFK, Lam LCW. Relevance of outcome measures in different cultural groups—does one size fit all? Int Psychogeriatr. 2007;19:457-66.

33. Binetti G, Mega MS, Magni E, Padovani A, Rozzini L, Bianchetti A, et al. Behavioral disorders in Alzheimer disease: a transcultural perspective. Arch Neurol. 1998;55:539-44.

34. Chow TW, Liu CK, Fuh JL, Leung VPY, Tai CT, Chen LW, et al. Neuropsychiatric symptoms of Alzheimer's disease differ in Chinese and American patients. Int J Geriatr Psychiatry. 2002;17:22-8.

35. Truzzi A, Ulstein I, Valente L, Engelhardt E, Coutinho ESF, Laks J, Engedal K. Patterns of neuropsychiatric sub-syndromes in Brazilian and Norwegian patients with dementia. Int Psychogeriatr. 2013;25:228-35.

36. Salazar R, Dwivedi AK, Royall DR. Cross-ethnic differences in the severity of neuropsychiatric symptoms in persons with mild cognitive impairment and Alzheimer's disease. J Neuropsychiatry Clin Neurosci. 2017;29:13-21.

\section{Ready to submit your research? Choose BMC and benefit from:}

- fast, convenient online submission

- thorough peer review by experienced researchers in your field

- rapid publication on acceptance

- support for research data, including large and complex data types

- gold Open Access which fosters wider collaboration and increased citations

- maximum visibility for your research: over $100 \mathrm{M}$ website views per year

At BMC, research is always in progress.

Learn more biomedcentral.com/submissions 